\title{
INDUCTIVE AND CAPACITIVE ARRAY IMAGING OF BURIED OBJECTS
}

D. Schlicker ${ }^{1}$, A. Washabaugh ${ }^{1}$, I. Shay ${ }^{1}$, N. Goldfine $^{1}$

${ }^{1}$ JENTEK Sensors, Inc., Waltham, MA, USA

\begin{abstract}
Despite ongoing research and development efforts, landmine and unexploded ordnance infestation continues to be a problem. Remediation efforts typically utilize inexpensive handheld metal detectors that rely on the principle of electromagnetic induction. While these devices often detect all relevant metal objects, they generally cannot discriminate between harmful objects and harmless clutter. False indications continue to far outnumber verified detections. One promising method for improving the discrimination between harmful and harmless objects is the use of sensor arrays that provide images of hidden objects and complementary information about the buried object from a variety of angles.

This paper will review the progress of high resolution inductive and capacitive sensing array development for buried object detection. These sensor arrays use unique designs incorporating a single drive with multiple sense elements. The drive creates a shaped magnetic or electric field pattern that concentrates the field energy into longer wavelength spatial modes with "focused" penetration into the ground. Images obtained from scans over buried objects provide a basis for spatial filtering and signal processing while multiple sensor arrays placed at different positions within the drive provide different "views" of buried objects and clutter. Model-based grid measurement methods will also be reviewed as a real-time method for multiple property measurements, and real-time data analysis/image generation.
\end{abstract}

Introduction: The capability of existing technologies to discriminate between unexploded ordnance (UXO), land mines and harmless clutter continues to limit the efficacy of site remediation. Inductive sensors with a single sensing coil are commonly used for detection. These single coil methods often detect all relevant metal objects but generally cannot discriminate between harmful objects and harmless clutter. Also, research into model based methods, using such single coil sensors, to discriminate between harmful objects and clutter have shown limited success when variations in the object orientation, material types, and damage are considered, even if clutter was not a problem. False indications continue to far outnumber verified detections and new methods are needed to provide a fieldable detection and clutter suppression capability.

An approach that addresses this need is to both increase the resolution of images so that information can be obtained about object shape and orientation and to design sensors that provide predictable responses. As an example, shaped field drives can be used to create a magnetic or electric field distribution with an intentional shape and to interrogate hidden objects and clutter at varied depths, with varied field orientations, with varied relative positions of small sensing coils to the buried object, and with the sensing elements and object at varied positions within the applied field. Predictable responses permit the use of models derived from basic physical principles to support inversion algorithms and to characterize object properties. This approach is consistent with ongoing development efforts validating both the use of multiple sense element locations and orientation relative to hidden objects [1] and the use of model based approaches to discriminate between objects [5].

Shaped-field sensor arrays that permit both high resolution imaging and accurate modeling of the sensor response to buried objects, developed by JENTEK, are based on unique designs that use a single drive winding or electrode and multiple sense elements. For metal objects, the drive winding is driven by an electric current to create a magnetic field and the response to the objects is detected through electromagnetic induction. For low or no-metal objects such as plastic land mines, a drive electrode is driven by an electric potential to create an electric field and the object response is detected through capacitive coupling.

An example inductive sensor array, called an $\mathrm{MWM}^{\circledR}$-Array, is illustrated in Figure 1. The drive creates a shaped magnetic field pattern, which provides a continuous variation in the orientation of the magnetic field relative to the buried ordnance. The magnetic field shape is created by the distribution of current in the drive winding segments. Arrays of small inductive coils or other sense elements placed throughout the shaped field measure the magnetic field variations caused by conducting or magnetic UXO and clutter. Images obtained from scans over buried objects provide a basis for spatial filtering and signal processing, with a goal to achieve enhanced detection and clutter suppression by discriminating between objects based on shape, through high resolution imaging, and object properties, through model based analysis. A similar approach is followed for the capacitive sensor array shown in Figure 10. 
Figure 2 illustrates how the depth of penetration of the magnetic field into the ground depends upon the spatial wavelength $\lambda$ of the winding currents, the excitation frequency $f$, and the ground properties in terms of the electrical conductivity $\sigma$ and magnetic permeability $\mu$. The depth of penetration is limited at low frequencies by the spatial distribution of the winding current and at high frequencies by the skin depth $(\delta=1 / \sqrt{\pi f \mu \sigma})$. The depth of sensitivity is a similar concept to the depth of penetration and reflects the measurable signal level for a specific object (geometry, orientation, and material properties) in a specific material (e.g., soil) relative to the measurement noise (from both the instrument and material inhomogeneities). An advantage of this approach is that different spatial wavelength drive windings have different depths of sensitivity (at the low frequencies) which permits depth profiling so that the properties of objects at different depths can be determined.
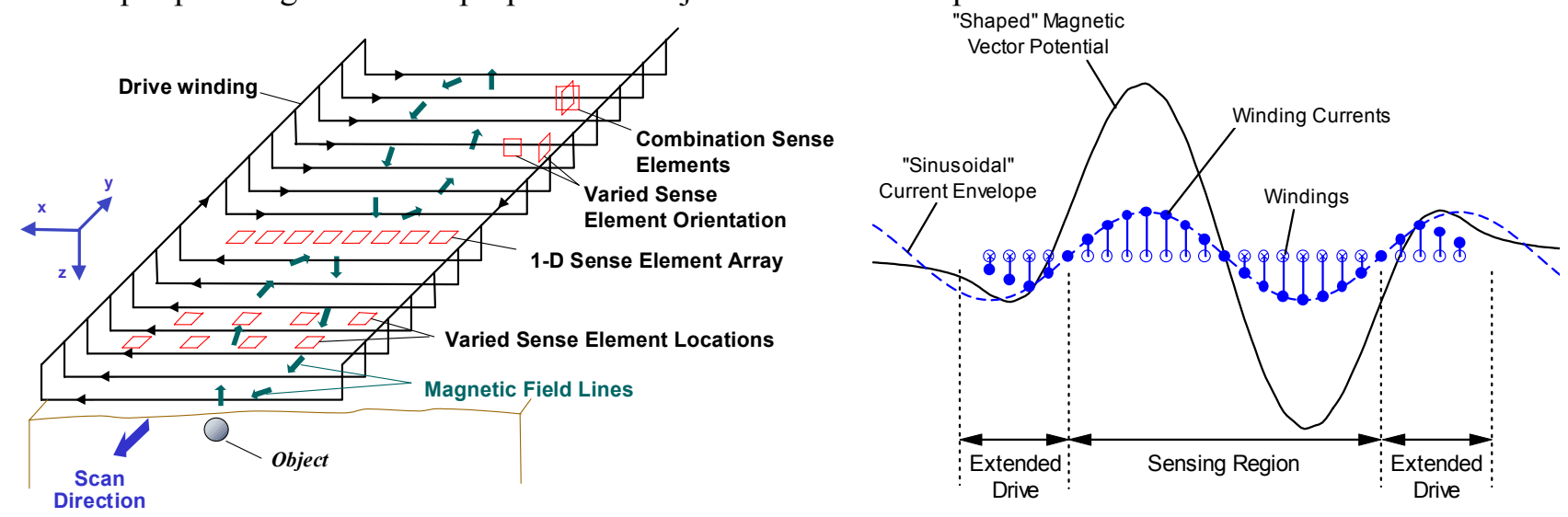

Figure 1. (left) Schematic for a distributed drive winding with multiple sense elements. (right) Diagram of the drive winding pattern and sinusoidal "shaped" field drive.
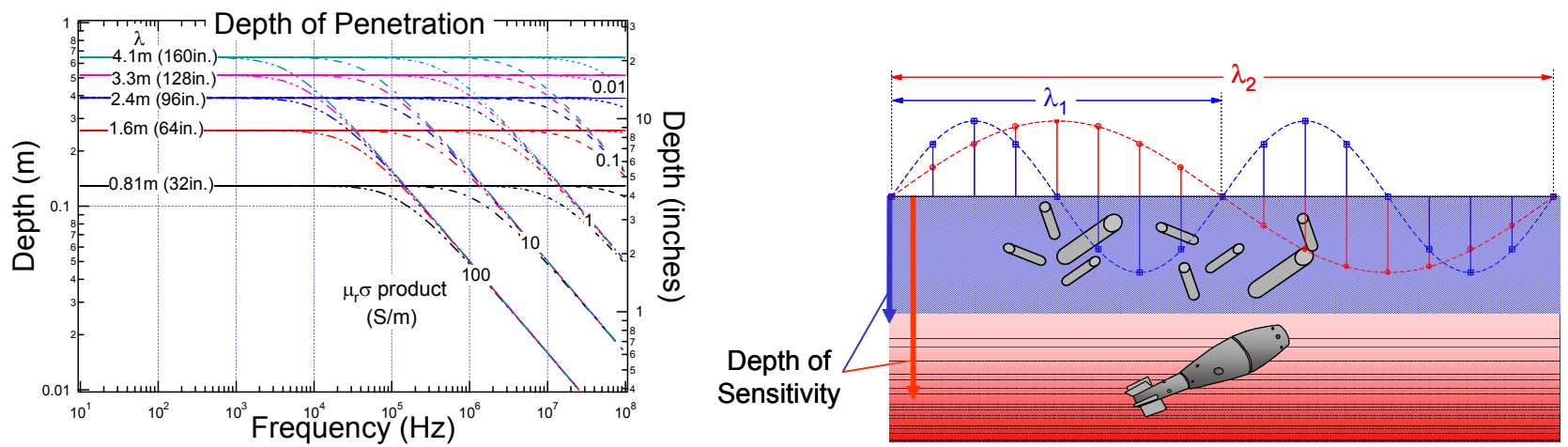

Figure 2. (left) Magnetic field depth of penetration dependence on sensor geometry, excitation frequency, and ground properties, and (right) schematic for sensor geometry effect on depth of sensitivity.

An eight-element prototype $\mathrm{MWM}^{\circledR}$-Array developed several years ago demonstrated the capability to produce images of buried ordnance $[3,4]$. Although this array can already provide a much higher resolution than single coil methods, recent developments of JENTEK's impedance measurement instrumentation permit data acquisition of many (up to 39) channels in parallel so that more sensing elements can be monitored continuously. This increased number of sense elements can be used to both increase the spatial resolution of the scan images and to provide complementary information about the object properties by placing sense elements at different positions and/or orientations within the footprint of the array. Consequently, a single linear array of 16 sensing elements has been fabricated [2]. The sense elements in the array are $1.3 \mathrm{~cm}$ diameter coils. The center-to-center spacing is 2.54 $\mathrm{cm}$. The array is designed to fit within the $0.6 \mathrm{~m}$ width of the drive winding structure with a reasonable spacing between the ends of the array and the return/side portions of the drive winding. The distance to the return portions of the drive winding needs to be large to minimize extra coupling of the magnetic field between the drive winding and the sense coils since this extra coupling is a source of element-to-element variability for the array response. 
Part of the motivation for the design of the MWM-Array is to have the capability to accurately model the response of the sensor to detected objects. These modeling efforts have focused on improved representation of the drive windings and the interactions of multiple objects. This new model not only accounts for the drive winding enhancement, but also includes the effects of the return windings not addressed in our previous model. The modeling approach follows the approach of Braunisch et al for distributions of conducting and permeable bodies of generic geometry [5]. The model uses the magnetic fields created by the drive winding structure to calculate the secondary dipole fields excited for objects, which can be represented by a polarization tensor, and any interactions between multiple objects. Spherical objects have been specifically used in initial modeling and experiments. The sensing element response is calculated from the secondary fields for spheres of various diameters, positions (depth and translation) and electrical properties (conductivity and permeability). Responses for spheres of fixed electrical properties located directly over the sensing element at varying depth and with varying diameter have been tabulated into depth-diameter measurement grids, allowing quick inversion from measured sensing element response (transimpedance) to depth-diameter parameters. This patented "grid method" supports rapid (real-time) object identification and discrimination [6,7].

Results and Discussion: Representative scan images over aluminum spheres are shown in Figure 3 . These images show the raw data in the form of the magnitude of the signal for each element. Note that complementary images can also be created for the real (in-phase) and imaginary (quadrature phase) parts of the complex impedance. Although the color scales are different in each case, the spheres are readily discerned from any background variations. These measurements also demonstrate the diffusion or spreading of the field response as objects get farther away from the sensor array. The smaller but deeper sphere provides a larger spatial image than the larger but shallower sphere. Thus, the image by itself cannot be used to determine the object size and other information, such as the magnitude scaling, is necessary to correctly differentiate the object properties. Alternatively, if the depth is known, then the image size directly reflects the object size.

Scan images from the array can also reflect the orientation of hidden objects. To demonstrate this effect, $0.9 \mathrm{~m}$ long pipes were placed at various orientations and scanned with the array. A representative set of measurement images is shown in Figure 4 for a $3.8 \mathrm{~cm}$ diameter pipe oriented perpendicularly and at 45 degrees to the sense element array. Similar images are obtained with larger pipe diameters and, like the spheres, the spatial width of the images increases with object depth and size.

(a)
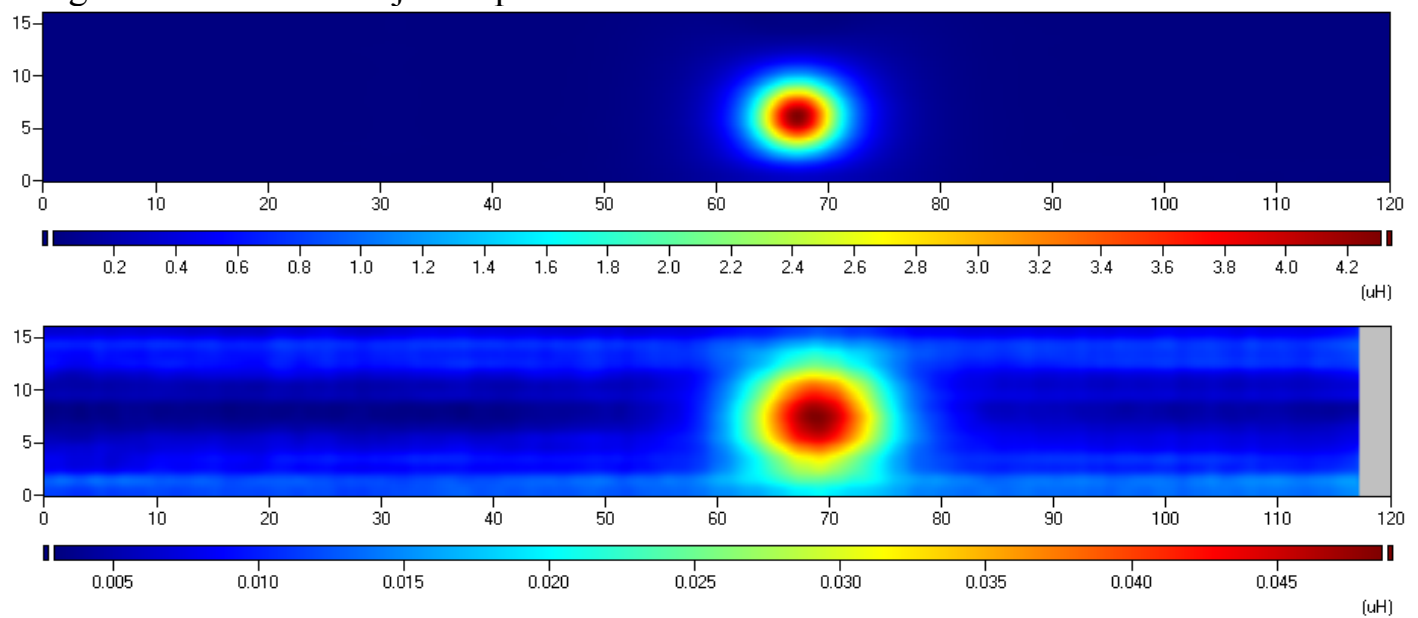

(b)

Figure 3. Array magnitude response over (a) a $12.7 \mathrm{~cm}$ diameter aluminum sphere at a depth of $5 \mathrm{~cm}$ and (b) a 6.4 $\mathrm{cm}$ diameter aluminum sphere at a depth of $20 \mathrm{~cm}$. 


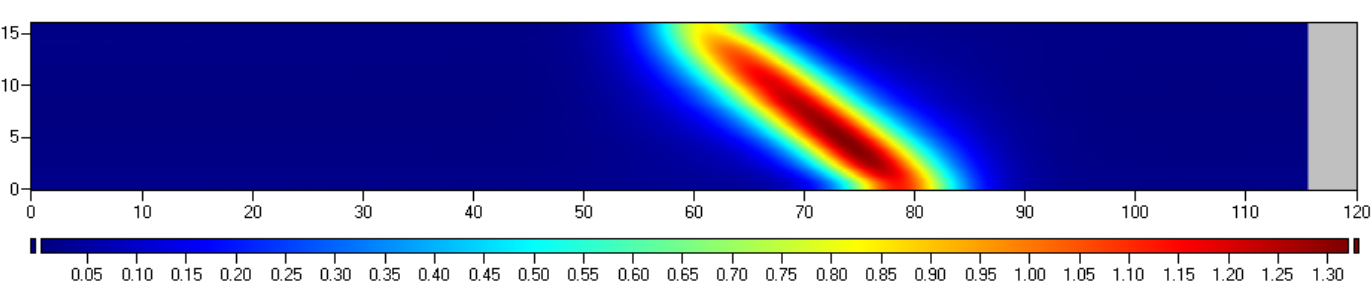

(a)

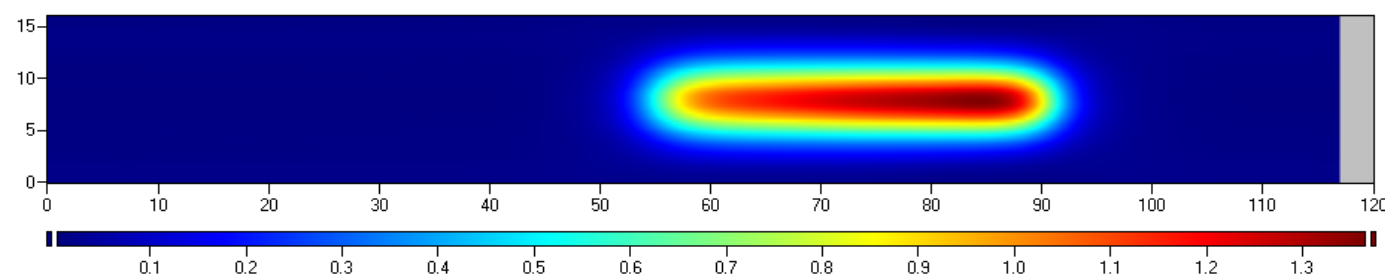

(b)

Figure 4. Array magnitude response over a $91 \mathrm{~cm}$ long $3.8 \mathrm{~cm}$ diameter pipe at a depth of $10 \mathrm{~cm}$ oriented (a) at 45 degrees to the sense element array and (b) perpendicular to the sense element array.

While images can provide some information about the shape, size and orientation of a buried object, models for the sensor response can be used to provide information about the properties of the object. A convenient method for converting the measurement data into physical properties in real-time is to use measurement grid methods. This patented method uses a database of responses, generated prior to data acquisition, that relates the sensor response to variations in object material properties, size, depth, orientation, etc. over the range of interest. Two-dimensional subsets of the database can be visualized as a measurement grid that relates two measured parameters, such as the magnitude and phase of the transimpedance, to two unknown parameters of interest, such as the size and depth of an object. If additional properties are to be determined, such as the electrical conductivity or shape of the buried object, additional information (e.g., from multiple frequency measurements, from responses from sense elements not directly over the object, or from the scan images) and higher order (e.g., three or more dimensional) databases need to be used.

Figure 5 shows a representative measurement grid for aluminum spheres along with the measurement response as different size aluminum spheres are lowered beneath the center sense element (element 8 ). The model was used to calculate an appropriate measurement grid for the properties of the sphere (e.g., magnetic permeability and electrical conductivity) and for the sense element position and orientation within the drive winding. In this case, the measurement grid is plotted as the imaginary part of the signal against the real part of the signal, where the signal is expressed in terms of the magnetic field intensity $H$ relative to the drive current $I$. When the sense element is directly over the object the measurement grid indicates reasonable sensitivity to the size and depth of the object, as indicated by the relatively large spacing between the grid lines. For each sphere, the model and data show a nonlinear response and the data follows a line of constant size as the depth is varied. The measurement grid can be used in conjunction with the image scans to determine effective properties for hidden objects, as illustrated in the right side of Figure 5. The data points were taken from the peaks in the images in the real and imaginary components of the impedance. This data compares favorably with the measurements taken as the aluminum sphere was lowered by hand beneath the center sense element.

Figure 6 shows the estimated properties obtained from the center sense element as aluminum spheres of different size are lowered beneath it. The depth estimates are generally within approximately $2 \mathrm{~cm}$ to a depth of approximately $20 \mathrm{~cm}$, which is where the noise in the measurements causes some of the data to fall off of the measurement grid and the standard deviation of the measurement, indicated by the vertical error bars, increases substantially. The associated estimates of the sphere sizes are in reasonable agreement with the known sphere sizes for shallow depths. These estimates become less reliable at depths greater than approximately $20 \mathrm{~cm}$ because of the decay of the imposed magnetic field intensity with depth. Use of a larger drive winding wavelength would reduce this decay. Also, better size and depth estimates at deeper depths are possible with the larger objects. Similar property estimates can be performed even when the object is not under the sense element. Figure 7 shows 
representative measurement grids for several sense elements in the array when a sphere is beneath the center sense element. Nearby sense elements can be used to provide complementary information about the buried object, but the grids tend to collapse and become smaller as the object is positioned farther away, which indicates a reduction in sensitivity to object properties.
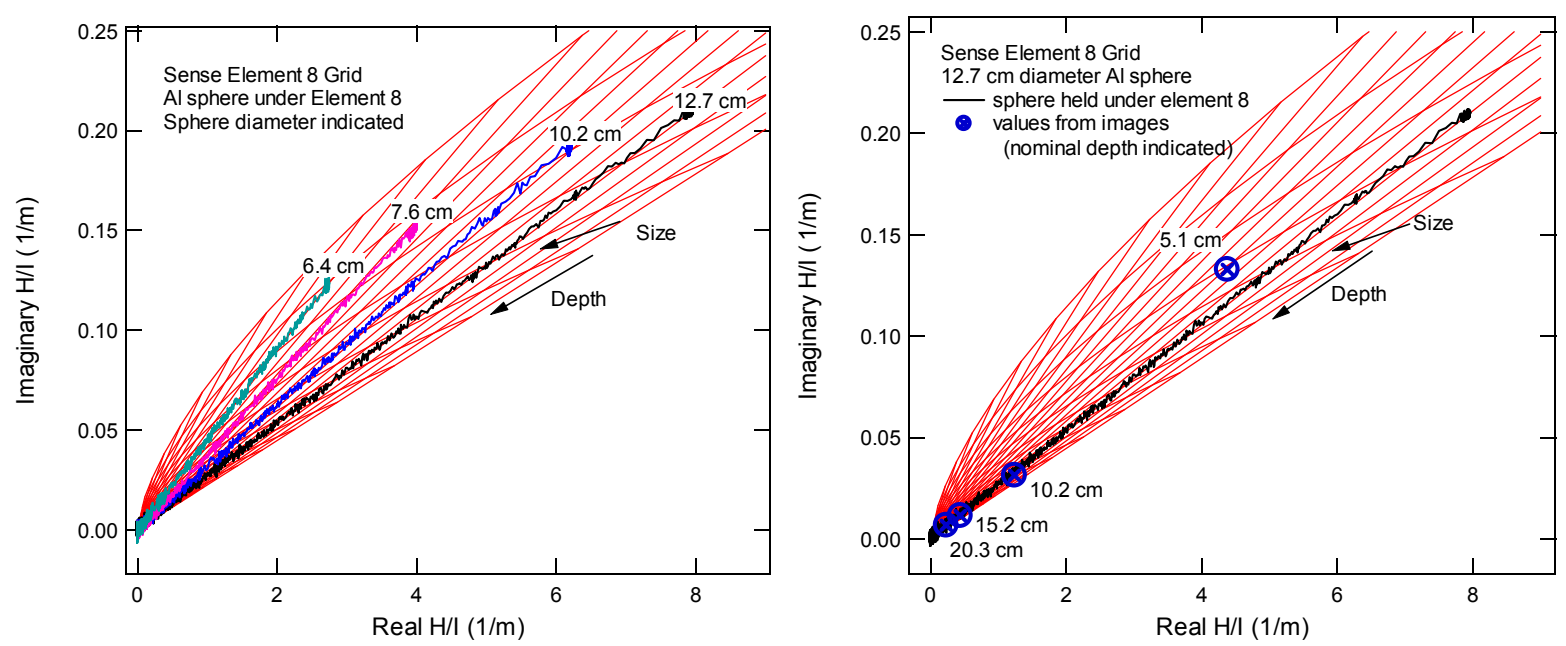

Figure 5. (left) Representative diameter-depth grid and representative data for aluminum spheres under the center sense element (Element 8). (right) Response of the center sense element to a $12.7 \mathrm{~cm}$ diameter aluminum sphere. The stationary cart data, where the sphere was held beneath the sense element and lowered by hand, is plotted along with impedance values taken from the peak signal responses in the image scans.
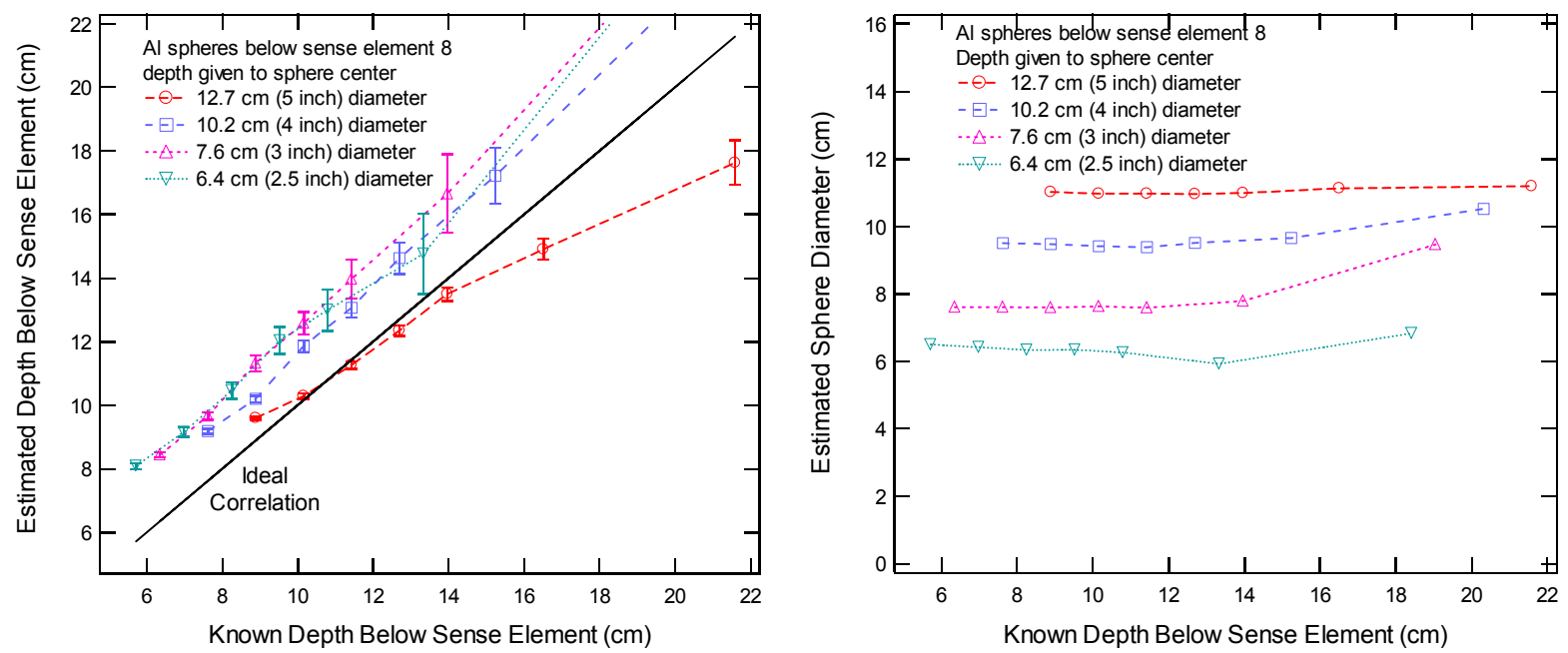

Figure 6. Estimated depth (left) and size (right) for aluminum spheres placed beneath the center sense element.
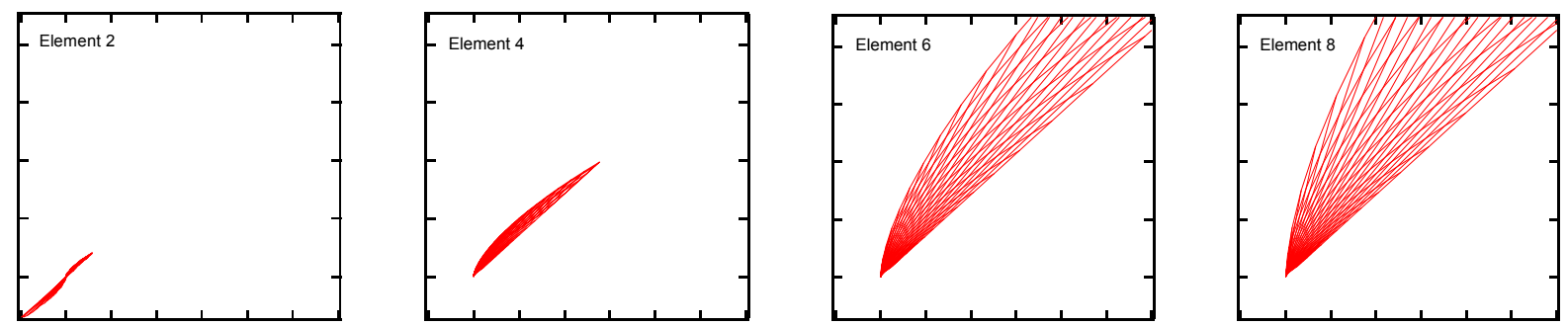

Figure 7. Size-depth measurement grids for several sense elements (elements 2, 4, 6, 8) when an aluminum sphere is beneath the center sense element (element 8), with the same scale used for all of the plots. 
Measurements of objects beneath a sense element as the object depth is varied also indicate that the real and imaginary parts of the signal can be used to discriminate between objects. Figure 8 shows the response as the depth of several UXO is varied from approximately $3.8 \mathrm{~cm}$ from the array to the top of the object, to greater than $30.5 \mathrm{~cm}$. Except for the spherical BLU-26 the response was measured for several orientations of the major axis (longest dimension) either parallel or perpendicular to the linear array of sense elements, or vertical with the UXO pointed down (tail up). In each case, the response converges to the air point when the object is sufficiently far from the array to be detected. For the M385, all three orientations provide essentially the same response, with a large real component and a small imaginary component. For the BLU-26, the real and imaginary components are comparable. For the higher aspect ratio M86 and the MK2, the parallel and perpendicular orientations are similar but the vertical orientation provides a noticeable shift in the response. The response shift is larger for the larger M86 UXO. Some of the reduction in signal associated with the vertical orientation is due to the center of the object being effectively deeper for the vertical orientation. The similarities in the responses for the M86 and MK2, at least in the parallel and perpendicular orientations, along the depth variation line indicates potential non-uniqueness issues for a single sense element and a simple sphere model response; the objects give a similar response at different depths, even though they are different sizes. Other information, for example from other sense elements (e.g., images), other sensing element orientations, or multiple frequencies, would be needed to properly discriminate between these objects.

Measurements have also been performed over multiple objects in order to show the benefit of high resolution imaging. Figure 9 shows several response images for the bomblets aligned parallel to the sense element array. At shallow depths and with large separation distances, the response of each bomblet is distinct. For deeper depths and smaller separation distances, the interactions between the objects become more apparent and the diffusion of the magnetic field response leads to an overlap of the effective response and a "single" object response. This response has an effective orientation associated with alignment of the objects and also local maxima that are associated with the center of the objects. For very deep objects and for very small separations, the effective orientation of the response becomes less apparent and symmetric responses result. The use of a high resolution imaging array allows the spatial variations of the response from the objects to be captured, including orientation and local maxima and minima in the response, that may permit separation of object responses.
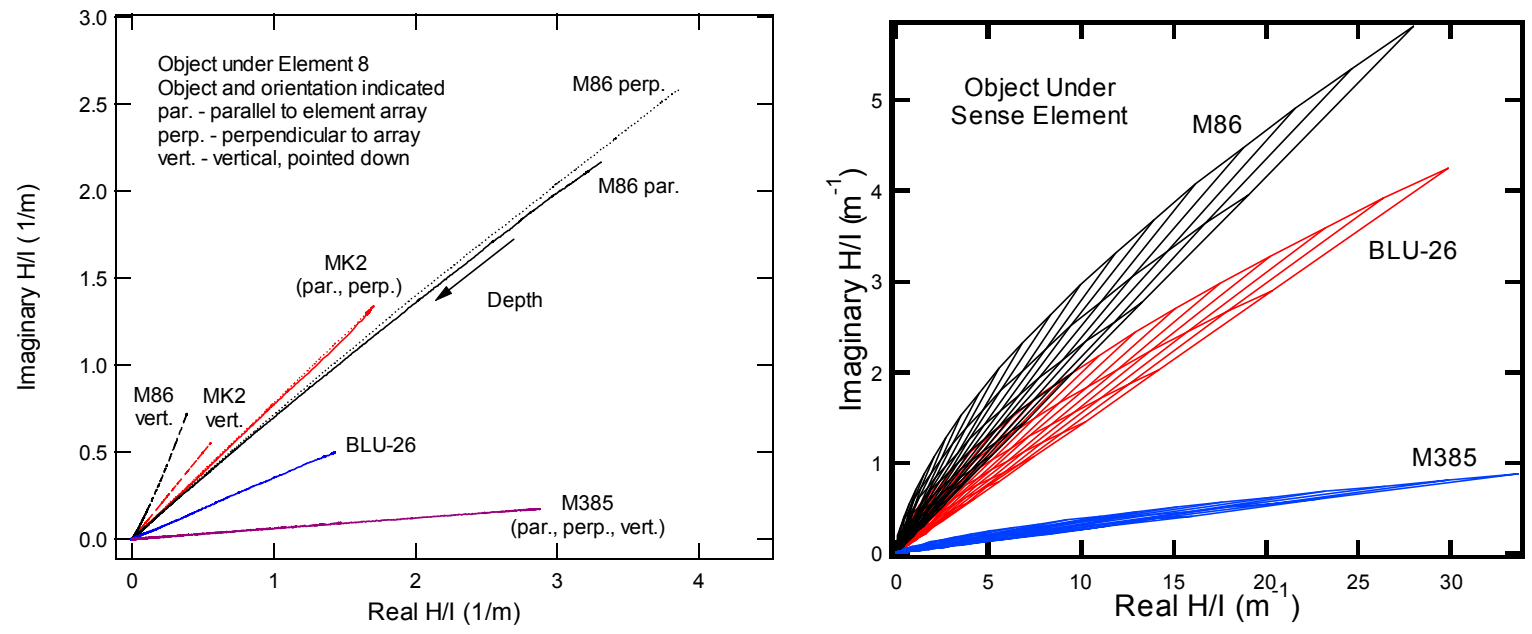

Figure 8. (left) Response as the depth of several objects beneath a sense element is varied. (right) Representative measurement grids for objects having different material properties. 

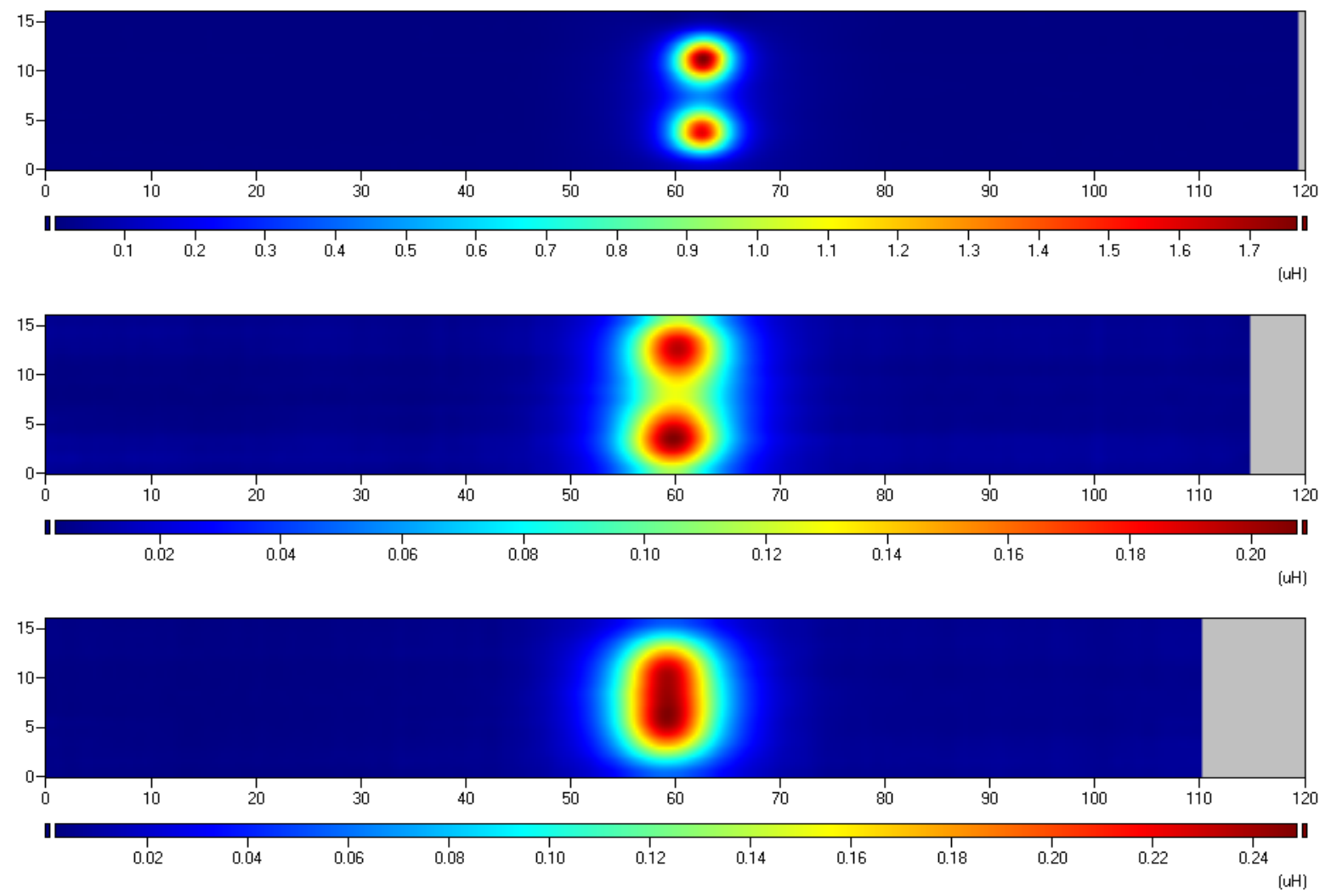

Figure 9. Response of two BLU-26 bomblets oriented parallel to the sensor array. (top) $20.3 \mathrm{~cm}$ apart and $3.8 \mathrm{~cm}$ deep. (middle) $20.3 \mathrm{~cm}$ apart and $11.4 \mathrm{~cm}$ deep. (bottom) $10.2 \mathrm{~cm}$ apart and $11.4 \mathrm{~cm}$ deep.

As a demonstration of the imaging capability of a five-element capacitive array, measurements were performed with low-metal content landmines buried in a laboratory sandbox. Figure 10 shows a schematic of the sensor array and images for an M14 landmine buried at several depths, with sensitivity to the presence of the landmine lost at a depth of approximately $3 \mathrm{~cm}$. With a nominal air gap liftoff distance of $2 \mathrm{~cm}$, the total depth of sensitivity is approximately $5 \mathrm{~cm}$ below the sensor, which is consistent with the approximation that dielectric sensors have a depth of sensitivity of roughly one-quarter of a wavelength, which is $20 \mathrm{~cm}$ for this capacitive sensor.

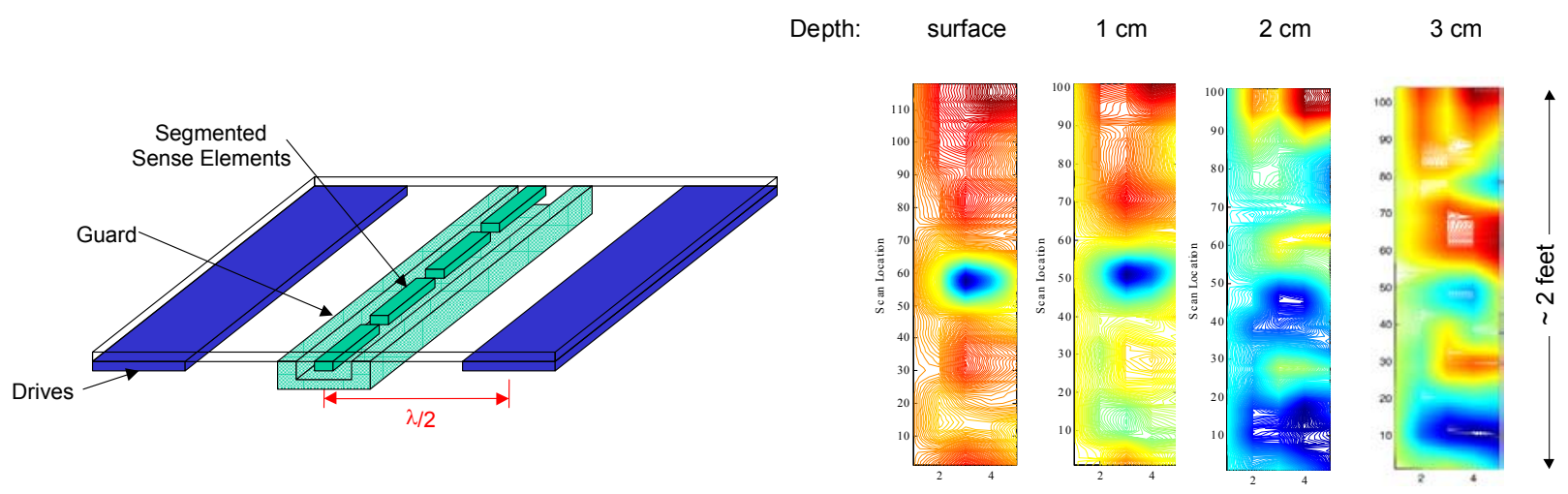

Figure 10. (left) A capacitive sensor array and (right) scan images of a capacitive sensing array over an M14 landmine buried at several depths. 
Conclusions: This paper presented some results from ongoing efforts to develop a practical field instrument for the detection and discrimination of buried objects. In particular, the use of a high resolution inductive array of sensing elements allows information about object shape and orientation, and even the presence of multiple objects, to be readily discerned. The use of models also permits the inverting of the measurement data into meaningful property estimates, but additional work is required to provide a robust discrimination method. The model has been shown to capture the basic response of the sensors to spheres under the footprint of the array and with various positions of the sphere relative to the sense elements. Each of the sense elements provide information about the object properties; the capability for using this information is related to the sensitivity, which can be determined from the model, and the measurement noise, which is determined empirically. A robust method of discrimination is expected to combine data from multiple sense elements and multiple sense element orientations, along with multiple excitation frequencies and drive wavelengths.

\section{References:}

1. Becker, A. "Detection of Classification of Buried Metallic Objects," SERDP Project UX-1225, presented at the Partners in Environmental Technology Symposium and Workshop, Washington, D.C., December, 2002.

2. Goldfine, N., Washabaugh, A., Schlicker, D., Shay, I., "High Resolution Inductive Sensor Arrays for UXO Detection, Identification, and Clutter Suppression," SPIE AeroSense 2003, Orlando, FL, April 21-25, 2003.

3. Goldfine, N., Washabaugh, A., Schlicker, D., "High Resolution Inductive Array Imaging of Buried Objects," SPIE AeroSense 2000, Orlando, FL, April 24-27, 2000.

4. Goldfine, N., Schlicker, D., Washabaugh, A., Clark, D., Zahn, M.., "New Quasistatic Magnetic and Electric Field Imaging Arrays and Algorithms for Object Detection, Identification, and Discrimination," SPIE AeroSense, Orlando, FL, April 5-9, 1999.

5. Braunisch, H., Ao, C.O., O’Neill, K., Kong, J.A. “Magnetoquasistatic Response of a Distibution of Small Conducting and Permeable Objects," Geoscience and Remote Sensing Symposium, Proceedings, vol. 4, 2000, pp. 1424-1426.

6. Goldfine, N.J., "Apparatus and Methods for Obtaining Increased, Selectivity and Dynamic Range in Property Measurement Using Magnetometers," U.S. Patent Number 5,629,621.

7. Goldfine, N.J., et al., "Magnetometer with Waveform Shaping," U.S. Patent Number 6,144,206. 